\title{
Article
}

\section{Great expectations? A qualitative study of health professionals' perspectives on breaking bad news about rehabilitation potential after traumatic brain injury or spinal injury}

Peel, Rosemary, Jones, Stephanie, Miller, Colette and Gibson, Josephine

Available at http://clok.uclan.ac.uk/26401/

Peel, Rosemary ORCID: 0000-0003-0784-9392, Jones, Stephanie ORCID: 00000001-9149-8606, Miller, Colette ORCID: 0000-0003-0620-6029 and Gibson, Josephine ORCID: 0000-0002-3051-1237 (2020) Great expectations? A qualitative study of health professionals' perspectives on breaking bad news about rehabilitation potential after traumatic brain injury or spinal injury.

Disability and Rehabilitation, 42 (22). pp. 3209-3214. ISSN 0963-8288

It is advisable to refer to the publisher's version if you intend to cite from the work. http://dx.doi.org/10.1080/09638288.2019.1589586

For more information about UCLan's research in this area go to http://www.uclan.ac.uk/researchgroups/ and search for <name of research Group>.

For information about Research generally at UCLan please go to http://www.uclan.ac.uk/research/

All outputs in CLoK are protected by Intellectual Property Rights law, including Copyright law. Copyright, IPR and Moral Rights for the works on this site are retained by the individual authors and/or other copyright owners. Terms and conditions for use of this material are defined in the policies page. 


\section{Abstract}

Background: Neurorehabilitation units play an important role in facilitating recovery for those with complex needs following a neurological event. National guidance highlights the importance of providing patients and their families with information and fostering realistic expectations. This may involve the breaking of bad news. The aim of this study is to explore health professionals' perspectives on breaking bad news in the neurorehabilitation setting. Method: 15 health professionals (physiotherapists, occupational therapists, nurses, speech therapists, psychologists and doctors) working at a 24 bedded neurorehabilitation unit in a National Health Service acute trust in England were recruited. A qualitative study was conducted using patient vignettes to facilitate discussions during semi-structured interviews and a focus group. The results were analysed using thematic analysis.

Results: Four major themes emerged: influencing factors, current approaches used, staff experiences, and strategies to improve breaking bad news. There was a need for better management of patients' and families' expectations. Breaking bad news was seen as emotionally demanding yet often unrecognised work.

Conclusions: Breaking bad news in the neurorehabilitation setting is complex and underrecognised work, involving multiple health professionals. There is a need for both experience and training to improve skills and confidence in breaking bad news.

Keywords: Multidisciplinary Team, Communication, Neurology, Neurorehabilitation, Expectation Management. 


\section{Introduction}

Neurological trauma, such as traumatic spinal cord or brain injury, is a leading cause of adult acquired disability. It is frequently life-changing for the patient and their family. Inpatient neurorehabilitation units play an important role in facilitating recovery and optimising independence for those with complex needs following a neurological event. Needs are complex because of the level of disability caused, and may include any combination of medical, physical, cognitive, communication, behavioural or social needs [1]. Patients admitted to these specialist units, and their families, may have high expectations of the rehabilitation process and the level of recovery that will be achieved during their stay; others may have unrealistically pessimistic expectations.

Neurorehabilitation is recognised as a complex process and often necessitates a protracted stay on a specialist unit. National guidance [1] highlights the importance of providing patients and their families with information and fostering realistic expectations. Clear communication at every stage of rehabilitation is vital $[2,3]$. To support communication there are many conversations between patients, family members, and staff in the neurorehabilitation unit setting that involve sharing information about rehabilitation potential [1]. It is recognised that health professionals other than doctors are involved in the delivery of bad news [4].

Information sharing should promote the development of realistic expectations for recovery, but these conversations can be difficult for all parties involved. They may have a negative impact on an individual's and their family's expectations and perceptions about their future [5]. This type of information sharing is well-recognised in the context of life-threatening conditions or end of life care, and is defined as a health professional conveying perceived bad news to a patient or family, at a given point in time [6]. There are several approaches that health professionals can use to structure difficult conversations, such as the use of the 
'S-P-I-K-E-S' framework (Setting, Perception, Information, Knowledge, Empathy and Summarising) in oncology [6]. However, there is a lack of evidence on this topic specific to rehabilitation for life-changing, rather than life-threatening, conditions. Such conversations may be challenging, uncomfortable or unsatisfactory for staff, patients and family members [4,6-13]. To compound the situation further, it is often very difficult for healthcare professionals to accurately predict rehabilitation potential [8]. However, it is thought that well communicated information about rehabilitation potential can aid recovery and future well-being [14]. The support of the patient's family has also been shown to be important in the process of delivering bad news [15].

Information giving and expectation management are an important part of staff-patient communication in the neurorehabilitation unit setting and, because of the nature of patients' disabilities, this regularly involves sharing information which may be perceived as bad news [1]. There is, however limited research about the experiences of members of the multidisciplinary team sharing information about rehabilitation potential, particularly neurorehabilitation.

The study aimed to:

- Explore health professionals' lived experiences of having difficult conversations surrounding rehabilitation potential;

- Explore different strategies used to support these difficult conversations;

- Identify how future practice could be improved. 


\section{Method}

The study was conducted in a regional neurorehabilitation unit within an acute hospital within the United Kingdom National Health Service. Although only one unit was included this was fairly typical of neurorehabilitation units in England. The multidisciplinary team included physicians, nursing staff, occupational therapists, physiotherapists, psychologists and speech therapists. All registered health professionals working on the unit, from any professional background or length of experience, were invited to participate.

Staff were invited to take part in a group interview or an individual interview, depending on their preference and availability. The lead researcher, a member of the multidisciplinary team, approached other members of the team within the neurorehabilitation unit via e-mail, including an information sheet. Potential participants were given a minimum of 24 hours to decide if they wished to participate in the study. They were asked to contact the researcher in person, via email or telephone. A total of 15 participants responded from a team of around 30 health professionals.

The interviews were conducted in a quiet room at the neurorehabilitation unit. Participants were asked to complete a brief data collection form to capture demographic and clinical experience information. The focus group and the individual interviews were conducted by the researcher, followed the same semi-structured schedule, and were based on a discussion of two contrasting vignettes. Vignettes were used to stimulate discussion and minimise the risk of disclosure of information about specific patients. The vignettes were composed by the researcher, and were based on composites of patients who had received care on the neurorehabilitation unit, rather than on individual patients (Supplementary Information). The vignettes illustrated two contrasting 'difficult conversations' about rehabilitation potential, 
which might alter the patient's or family's expectations for recovery. The first vignette depicted a male with significant life-changing injuries. His family had unrealistically positive expectations of his recovery and had expressed hope that he could remain at the neurorehabilitation unit until improvement was seen. The team needed to break the news that his progress was likely to be limited, and that discharge planning needed to commence. In contrast, the second vignette illustrated a female patient who had made good progress in rehabilitation. Both the team and her family were keen to support her returning home, but she herself did not feel ready and was anxious about returning home due to unrealistically negative expectations.

Participants were asked about the factors that would influence their information sharing about rehabilitation potential, how they would manage the patient's and family's expectations in each scenario, and strategies they used to support potentially difficult conversations. The vignettes were then used to facilitate broader discussions using a semi-structured question schedule. The questions were neither definitive nor exhaustive.

An information sheet was given to all participants and written informed consent was obtained. Participants were informed that taking part was voluntary. Ground rules were set at the beginning of the group interview. Due to the nature of the group interview complete anonymity could not be guaranteed. Ethical approval was gained from the host academic institution, and research and development permission was obtained from the National Health Service hospital. Due to the small numbers of participants from some professional groups, each participant who was directly quoted in the findings was randomly allocated an initial letter rather than being identified by their profession, in order to minimise the possibility of 
direct quotes being attributed to individuals. Similarly, participants' level of experience in neurorehabilitation was divided into three categories.

The study utilised a phenomenological approach to explore the lived experience of staff working in this area of practice [16]. The focus groups and semi-structured interviews were digitally recorded and field notes were made, and the lead researcher also utilised a reflective diary. Digital recordings were transcribed verbatim and the audio recordings listened to for nuances such as tone of voice. The transcriptions, field notes and reflective diary were analysed, coding took place by hand using printed copies of the transcripts. Thematic analysis was used to analyse the transcripts [16]. Several sub themes were initially identified; these were clustered using an iterative approach to give four main themes. Confirmative and contradictory results were used to refine and condense the themes. A second researcher (SJ) independently coded the data for verification of the themes and codes and confirmed that the coding was a true reflection of the data captured with few examples of negative instances. Credibility was enhanced through member checking of the overall findings and themes, participants agreed the themes reflected their views and experiences; they also expressed relief that the issues they identified resonated with other staff. Participants were given the opportunity to respond to the researcher with any other comments, but no new comments were made. Whilst dependability of the data may vary based on staff changes following the research undertaken, no major service changes took place during or immediately following the study. 


\section{Results}

A total of 15 members of the multidisciplinary team participated; there were ten individual interviews and one group interview with five participants. The participants had varying levels of experience in neurorehabilitation, with five working at the neurorehabilitation unit for less than a year and four having more than 10 years' experience. Levels of experience were categorised as less than 2 years, between 2-5 years, and more than 5 years.' Participants included representatives from all the professions within the team (physicians, nurses, occupational therapists, physiotherapists, speech therapists, psychologists), three of the participants were male. In order to preserve anonymity, the exact details of profession and experience are not given. However, the participant quotes are accompanied by information about length of experience.

The group interview lasted approximately one hour, and individual interviews lasted between 20 and 45 minutes. Similar themes were identified from both the focus group and the interviews. Four main themes were developed: Factors that influence information sharing about rehabilitation potential; how staff initiate and conduct difficult conversations; lived experiences of information sharing about rehabilitation potential; and strategies to improve experiences of sharing perceived bad news (shown in table 1). The themes and sub-themes are explored in further detail below.

[Insert table 1 here] 


\section{Factors that influence information sharing about rehabilitation potential}

a) Understanding the patient's and family's expectations

Many participants described the patient's and family's pre-existing understanding of rehabilitation and recovery as having a strong influence on these difficult conversations:

Understanding where the family, and the patient are at that stage, and to acknowledge what their thoughts and expectations are... to gauge their understanding of the level of injury, the type of injury. To acknowledge the history that comes with the patient and what the family have experienced so far and seen in relation to the person's recovery, so I think being able to place this kind of breaking of news in context is really quite important. (Participant A, more than 5 years' experience)

Participants felt that patients' and families' expectations were influenced by numerous other factors, such as experiences in the acute phases of care whilst waiting for transfer to the neurorehabilitation unit, information from the internet, and discussions with other patients and families:

The patient's family go and read up on the internet, they speak to other patient's families outside the ward and, you know, because it is a loved member of their family they have their own expectations, sometimes all of these can be quite unrealistic. (Participant I, less than 2 years' experience)

\section{b) Staff expertise in neurorehabilitation}

Staff expertise, level of training, and length of experience in neurorehabilitation were thought to influence how and when information about rehabilitation potential was delivered: 
More senior staff who have been here longer are more able to identify that a patient won't go beyond transferring (i.e. will not walk again). But it's when you have less senior staff who don't have that experience, it's very hard for them to predict and manage the expectations because they don't know. (Participant B, more than 5 years' experience)

\section{c) Staff-patient-family relationships}

The relationship and rapport between staff, the patient and their family were described as important. Many participants said that it was vital to establish a good relationship involving clear communication and trust early in the rehabilitation process, partly because of the need for transfer of information early on, but also to convey the team's ethos and dedication:

[W]here possible to include the family at the earliest stage I think is, is crucial. (Participant A, more than 5 years' experience)

It comes down to trust, if the family see that this is a team that are actually busting a gut trying to do everything possible then they're more likely to accept that...If they understand your process of thinking and they trust that you are trying to do your best I think that it helps. (Participant $C$, more than 5 years' experience)

\section{How staff initiate and conduct difficult conversations}

a) Timing - 'drip-feeding'

There was divergence of opinion about optimum timing of these difficult conversations. A few participants felt that they should happen at a single point in time in a formal meeting, but most described a process of 'drip feeding' information in preparation for a structured meeting where the perceived bad news would be formally broken. This often involved inviting 
families into therapy sessions and then discussing the outcome of the session with them. This might facilitate acceptance and reduce emotional distress:

I don't think it will be a simple one-off meeting...I think at some stage there has to be a formal meeting, but I think you need to plan it as a process. (Participant D, more than 5 years' experience)

b) Clarity and consistency of approach to difficult conversations

When 'drip feeding' information, participants highlighted the importance of different team members using a consistent approach and language. Effective communication could support the patient and family to process the information, and could also facilitate improved acceptance of bad news. A clear, direct approach was also needed:

If they are hearing bad news, even if they have been drip fed the information in the past, I think the fact the language is kept consistent it gives more opportunity for that to be processed again. And giving time in that meeting for a little bit to be said, then processed, is important. (Participant E, between 2 and 5 years' experience)

I think it's about being clear in the things that you're saying 'cos sometimes you can put so much fluff around it that actually the actual message doesn't get through.

(Participant $F$, between 2 and 5 years' experience)

Some of the participants described real difficulties in fostering realistic expectations whilst trying to maintain hope: 
On one hand we want to give people hope but on the other we need people to be at least in the right frame of thinking. (Participant $C$, more than 5 years' experience)

Participants described a wish to prepare, for example through discussions with colleagues, before having these difficult conversations. They suggested that this should happen well before commencing discharge planning.

\section{c) Personnel involved in difficult conversations}

There was divergence of opinion about who was responsible for delivering perceived bad news, based on staff perceptions of how the family would accept the news, and the specific barriers to rehabilitation for the individual patient. Although some felt that physicians should take the lead role, others thought that therapists should lead because of their expert understanding of the patient's rehabilitation progress:

I think since we're the ones [therapists] who are assessing and have the understanding of it [rehabilitation]. I think we are the best, but I don't think people always listen to us. (Participant B, more than 5 years' experience)

Various factors influenced the chosen approach to sharing bad news; there was no single approach to these difficult conversations and each situation needed to be managed as an individual case and discussed by the multidisciplinary team. Although participants were asked about strategies they used to support sharing information with patients and families, many felt that the process defied any attempt to reduce it to a rigid formula: 
The best I can say is experience of what has happened in the past with different families, how that family have been reacting up to then, and hope that the gut feeling decision that is clinically reasoned is the correct one for that moment in time for that family. (Participant G, more than 5 years' experience)

I don't think there's necessarily a formula for that, but I think you might be able to identify key steps. (Participant D, more than 5 years' experience)

\section{Lived experiences of information sharing about rehabilitation potential}

Almost all the participants expressed some negative consequences of having these conversations in terms of personal and professional impact. These stressful and negative emotions could impact on professionals' personal well-being, could affect their therapeutic relationship with the patient, and might also reflect negatively on their self-perception of their skills as a therapist:

One particular case in the last few months that has kept me up at night, has had me worried sick about coming into work. Worried about how I was going to approach that family, how I was going to deal with it. (Participant H, more than 5 years' experience)

We are dealing with long term disability and we're almost dealing with the acute stages of anger and coming to terms, [it] can be really emotionally hard for the therapist as well. (Participant I, less than 2 years' experience) 
We feel responsible, particularly as a caring profession. Sometimes it's hard on us because we can't make them better. (Participant J, between 2 and 5 years' experience)

Although some experienced staff felt more confident, even they did not always feel sufficiently skilled to share perceived bad news about rehabilitation potential, and most participants felt underequipped to do this:

I think it's a skill and I think we're just assumed as therapists or clinicians that you just happen to have that, and it's not, it's a skill that needs to be taught and learned and practised. (Participant $F$, between 2 and 5 years' experience)

Some staff had the skills to competently break bad news, and rather than a lack of skill it was a lack of confidence that led them to feel underequipped:

Counselling or other kinds of therapy (skills) would certainly be beneficial, but I wouldn't want that necessarily to detract from the skills that other professionals already have...they're kind of core skills and maybe (the job of the psychologist) is to kind of reinforce a lot of the skills we all have as therapists. (Participant A, more than 5 years' experience)

It was also acknowledged that there were some positive experiences related to sharing information about rehabilitation potential that involved changing patient expectations, such as supporting patients to adjust and cope with their level of disability: 
We can talk in a positive manner, bearing in mind it's a devastating experience for them (patients and families) ... hope has been found to be therapeutic and powerful. (Participant A, more than 5 years' experience)

4.Strategies to improve experiences of sharing perceived bad news

a) Strategies for specific points in the patient's journey

Information provided during the early stages of recovery before transfer to the neurorehabilitation unit could influence expectations, but it was not easy to manage this. Suggested strategies included the provision of outreach services to patients awaiting transfer to the neurorehabilitation unit to support early rehabilitation and provide education, provision of written information to all patients referred to the neurorehabilitation unit, to support expectation management in the early stages, and the introduction of a key worker system.

Expectation management was unanimously described as vital at every stage of the rehabilitation process. It was a significant factor affecting when and how information about rehabilitation potential was discussed, and also influenced how the patient and family dealt with the information:

[I]t's about setting out what their expectations are at the beginning, so you need to listen to them, because their expectations may be very different from what we can actually offer. (Participant $F$, between 2 and 5 years' experience)

A strong theme was the importance of the patient and their family having access to the psychology service: 
Families can go and talk through their concerns and where they're at in terms of coming to terms with things with the psychology team and they are very expert, I think sometimes families need that. (Participant D, more than 5 years' experience)

It was also highlighted that giving perceived bad news about rehabilitation potential in the neurorehabilitation unit setting could influence long term acceptance by the patient and family of recovery and health provision:

[I]f we don't get this right at this stage potentially you have got a family in a situation that is antagonistic towards a health system for years and years to come. (Participant C, more than 5 years' experience)

\section{b) Professional development and support}

There was a clear emotional cost to sharing perceived bad news and having these difficult conversations, and participants expressed a need for emotional support and professional development strategies. Almost every participant expressed a wish to utilise the expertise within the team and develop some training to enhance their understanding of the grieving process and their skills in delivering bad news. However, given that skills in breaking bad news relied largely on exposure to similar situations and experiential learning, an experienced member of staff should take the lead role:

I think just the more you're exposed to it the potential - not to be easier - but the more natural it becomes. (Participant $K$, less than 2 years' experience) 
Participants also identified the importance of reflective practice in order to develop their skills and to manage the stressful nature of this work:

Sometimes just 'talking it out' gets it out of your system, so that is a good way of offloading all the emotional trauma that is building within us day in and day out from dealing with clients like this. It is also important to employ reflection in clinical practice, maybe write out something, maybe see how we could have done it differently. (Participant I, less than 2 years' experience)

Participants identified several areas for service improvement, including introduction of a keyworker system to support information giving and clear communication, development of written information to help foster realistic expectations early in the rehabilitation process, and the development of an outreach service to engage with patients and their families in the acute care setting before admission to the rehabilitation unit. Debriefing and reflective practice groups were proposed to support staff well-being. Training needs might be met by using the expertise within the team this included building staff confidence in having difficult conversations about rehabilitation potential. Access to evidence-based guidelines and other materials to support best practice was also suggested.

\section{Discussion}

This study suggests that sharing perceived bad news about rehabilitation potential in neurorehabilitation is a complex process. Participants felt some factors were more susceptible to influence, including expectations for service delivery. Other factors were seen as difficult to manage, including expectations formed in the acute stages of recovery. 
This study is one of the first to examine the process of 'breaking bad news' in the context of neurorehabilitation, and to examine the issue from a multidisciplinary perspective.

Limitations of the study include its use of staff from only one neurorehabilitation unit. The study site was familiar to the lead researcher, who worked as a member of the multidisciplinary team at the neurorehabilitation unit whilst conducting the research. This enabled in-depth understanding of the issues raised, but may also have influenced the recruitment of participants. Within the group setting it may have been difficult for participants to express conflict due to the researcher herself being a member of staff, and because the participants worked together regularly. This may have influenced the discussions.

The findings concur with previous research that breaking bad news is a complex process $[4,7,13]$ that can be unpredictable and challenging. Two major factors influencing the process were acceptance, and expectations for recovery. The process of recovery itself in relation to traumatic brain injury is complex, and this has an impact on the balance of realistic expectations with hope [17]. Much of the literature relating to breaking bad news is in relation to terminal diagnoses or in oncology $[5,6,9,10]$. Although there are some common factors and approaches to having these difficult conversations, it should be acknowledged that within rehabilitation the news is not usually life-threatening, but it is frequently lifechanging. Patients and families need to actively engage in order to adapt to a new future and way of living, and thus the fostering of hope is vitally important [14], for example families taking an active role in therapy and attending therapy sessions where possible. However, the difficulty of accurately predicting rehabilitation potential following brain injury, and the lengthy time of recovery [8], further complicate the process of discussing prognosis. 
The findings suggest that the approach to communication in the neurorehabilitation unit needs tailored to the individual needs of the patient and their family, and centred around trust and rapport $[2,3,7,18]$. Several participants described a need to be clear and succinct when sharing perceived bad news, and to use familiar language. This is supported by a widely used six-step approach to breaking bad news: 'S-P-I-K-E-S' (Setting, Perception, Information, Knowledge, Empathy and Summarising) [4]. This and other tools provide a structured step by step approach which may be helpful, but it is recognised there is also a need for experience, reflection, flexibility, and individualisation of the process.

Participants did not agree about which member or members of the multidisciplinary team should have the responsibility for sharing information about rehabilitation potential. There is a paucity of evidence on this topic. It is accepted by some researchers [10,11] that within stroke rehabilitation members of the multidisciplinary team will deliver bad news. However, it is not discussed who specifically should do this, and why. This area may warrant further research.

The findings also highlighted negative experiences of disclosing perceived bad news, and the impact on practitioners' emotional well-being [19]. This has been previously highlighted as a potential cause of work-related stress $[10,11,19]$. Both novice and experienced practitioners expressed relief their experiences resonated with others and expressed a desire for more formal and informal peer support to support their emotional well-being. They also expressed a strong desire for continuing professional development opportunities in this area. Although most of the available literature on strategies and approaches is aimed at medical staff, some elements may be useful for the multidisciplinary team in this setting. Reflective practice to 
promote learning and support, which has previously been utilised in stroke rehabilitation [11], may also be useful.

Health professionals want to provide support to patients and their families, but families in these situations have previously highlighted poor communication as a major issue [18]. Other than frameworks such as 'S-P-I-K-E-S' framework (Setting, Perception, Information, Knowledge, Empathy and Summarising) there is little literature to suggest best practice, particularly in neurorehabilitation. Families play a pivotal role in the recovery and ultimate well-being of patients with neurological trauma $[17,18]$, it may be beneficial to explore this topic further from the perspectives of patients and families.

\section{Conclusion}

All members of the multidisciplinary team in neurorehabilitation engage, to some extent in difficult conversations about rehabilitation potential. These conversations are a complex process, are affected by many factors such as patients' and families' expectations for recovery, and present many challenges for staff. It is important that realistic expectations are supported and fostered within neurorehabilitation, but further research into how this can be best achieved is needed. 


\section{References}

1. British Society of Rehabilitation Medicine. Specialised Neurorehabilitation Service Standards 7304 2015-PCATV2-forweb-4-5-16.doc Update 30.4.2015. [Cited 20 ${ }^{\text {th }}$ December 2017] Available from https://www.bsrm.org.uk/downloads/specialised-neurorehabilitation$\underline{\text { service-standards--7-30-4-2015-pcatv2-forweb-4-5-16.pdf }}$

2. Piccenna L, Lannin N A, Gruen R, et al. The experience of discharge for patients with a brain injury from the inpatient to the community setting: a qualitative review. Brain injury. $2016 ; 30: 3241-251$

3. Lefebvre H, Pelchat D, Levert M. Interdisciplinary Family Intervention Program: A Partnership Among Health Professionals, Traumatic Brain Injury Patients, and Caregiving Relatives. Int J Trauma Nurs. 2007; 14(2):100-113.

4. Warnock C. Breaking bad news: issues relating to nursing practice. Nurs Stand. 2014; 28(45): $51-58$

5. Randall T, Wearn A. Receiving bad news: patients with haematological cancer reflect on their experiences. Palliat Med. 2005; 19:594-601

6. Baile W, Buckman R, Lenzi R, et al. S-P-I-K-E-S a six-step protocol for delivering bad news: Application to the patient with cancer. Oncologist. 2000; 302-311 
7. Sexton D. As good as it's going to get. Bad news conversations in neurology: challenges for occupational therapists. Br J Occup Ther. 2013; 76:6

8. Lefebvre H, Levert M. Breaking the news of traumatic brain injury and incapacities. Brain Injury. 2006; 7: 711-718

9. Anderson S, Marlett N. Communication in stroke: the overlooked rehabilitation tool. Age Ageing. 2004; 33:440-443

10. Grainger K, Masterson S, Jennings M. 'Things aren't the same, are they?': The management of bad news delivery in the discourse of stroke care. Commun Med. 2005; 2(1):35-44

11. Phillips J, Kneebone I, Taverner B. Breaking bad news in stroke rehabilitation: a consultation with a community stroke team. Disabil Rehabil 2013; 325(7):694-701

12. Sparks L, Villigran M, Parker-Raley J, et al. A patient-centred approach to breaking bad news: Communication guidelines for health care providers. J App Commun Res 2007; 35(2):177-196

13. Fallowfield L, Jenkins V. Communicating sad, bad, and difficult news in medicine. The Lancet. 2004; 363 (9405): 312-319

14. Lohne L. Hope during the first months after acute spinal injury. J Adv Nurs. 2004; 47(3): 279-286 
15. Stejskal T. Removing barriers to rehabilitation: Theory-based family intervention in community settings after brain injury. NeuroRehabilitation. 2012; 31(1): 75-83

16. Silverman D. Interpreting qualitative data: $5^{\text {th }}$ ed. London: Sage Publications 2014.

17. Ruff R. Selecting the appropriate psychotherapies for individuals with traumatic brain injury: What works and what does not work? NeuroRehabilitation. 2013; 32(4):771-779

18.Foster AM, Armstrong J, Buckley A, et al. Encouraging family engagement in the rehabilitation process: a rehabilitation provider's development of support strategies for family members of people with traumatic brain injury. Disabil Rehabil. 2012; 34:1855-1862

19. Warnock C, Buchanan J, Tod AM. The difficulties experienced by nurses and healthcare staff involved in the process of breaking bad news. J Adv Nurs. 2017; 73(7), 1632-1645. 
Table 1 Themes identified in interviews

\begin{tabular}{|l|l|}
\hline Main Theme & Sub themes \\
\hline $\begin{array}{l}\text { 1. Factors that influence sharing } \\
\text { information about rehabilitation potential }\end{array}$ & $\begin{array}{r}\text { a. Understanding the patient's and } \\
\text { family's expectations }\end{array}$ \\
& $\begin{array}{r}\text { b. Staff expertise in neurorehabilitation } \\
\text { c. Staff-patient-family relationships }\end{array}$ \\
\hline $\begin{array}{l}\text { 2. How staff initiate and conduct difficult } \\
\text { conversations }\end{array}$ & $\begin{array}{l}\text { a. Timing - 'drip-feeding' } \\
\text { b. Clarity and consistency of approach to } \\
\text { difficult conversations }\end{array}$ \\
& $\begin{array}{l}\text { c. Personnel involved in difficult } \\
\text { conversations }\end{array}$ \\
\hline $\begin{array}{l}\text { 3. Lived experiences of information } \\
\text { sharing about rehabilitation potential }\end{array}$ & $\begin{array}{l}\text { a. Negative experiences } \\
\text { b. Positive experiences }\end{array}$ \\
\hline $\begin{array}{l}\text { 4. Strategies which may improve the } \\
\text { experience of sharing perceived bad news }\end{array}$ & a. Strategies for specific points in the \\
& batient's journey \\
&
\end{tabular}

